\title{
Dissipation of Spin Angular Momentum in Magnetic Switching
}

\author{
C. Stamm, ${ }^{*}$ I. Tudosa, H. C. Siegmann, and J. Stöhr ${ }^{\dagger}$ \\ Stanford Linear Accelerator Center, P.O. Box 20450, Stanford, California 94309, USA
}

A. Yu. Dobin

Seagate Technology LLC, Fremont, California 94538, USA

G. Woltersdorf ${ }^{\star}$ and B. Heinrich

Simon Fraser University, 8888 University Drive, Burnaby, British Columbia V5A 1S6, Canada

\author{
A. Vaterlaus \\ Laboratory for Solid State Physics, Swiss Federal Institute of Technology, 8049 Zürich, Switzerland
}

(Received 18 November 2004; published 19 May 2005)

\begin{abstract}
Applying one ultrashort magnetic field pulse, we observe up to 10 precessional switches of the magnetization direction in single crystalline Fe films of 10 and 15 atomic layers. We find that the rate at which angular momentum is dissipated in uniform large angle spin precession increases with time and film thickness, surpassing the intrinsic ferromagnetic resonance spin lattice relaxation of Fe by nearly an order of magnitude.
\end{abstract}

DOI: 10.1103/PhysRevLett.94.197603

PACS numbers: 76.90.+d, 75.30.Ds, 75.70.Ak

The bottleneck of magnetization dynamics is established by the necessity to conserve angular momentum whenever the magnetization $\vec{M}$ changes direction or magnitude. After an external excitation the spin system will ultimately equilibrate with the lattice. An equilibration time of the order $10^{-10} \mathrm{~s}$ is obtained from the line width of ferromagnetic resonance (FMR). Experiments on the technically important precessional reversal of $\vec{M}$ are successfully simulated by inserting the FMR derived dissipation into the Landau-Lifshitz-Gilbert (LLG) equation [1-4]. Yet the fundamental question in spintronics is about faster processes, resulting in temporary energy and angular momentum storage before their ultimate dissipation to the lattice. Two such processes have been considered. One is the dissipation within the spin system itself [5], the other is the possibility of transfer of angular momentum from the spin to the orbital degrees of freedom in pulsed laser excitation [6]. This latter process can lead to ultrafast $(\approx$ $1 \mathrm{ps})$ dissipation.

Applying the fastest conventional magnetic field pulses of $\approx 10^{4} \mathrm{~A} / \mathrm{m}$ amplitude and $\approx 100$ ps duration [1-5], $\vec{M}$ will reverse its direction once performing a complex motion induced by the simultaneous action of the pulse and the anisotropy fields. Here, we initiate the dynamics by moving $\vec{M}$ into a nonequilibrium position with an uniquely fast and powerful magnetic field pulse [7-9] and later look into which of the two possible equilibrium positions $\vec{M}$ has relaxed. A large magnetic pattern, revealed by magnetic microscopy, is generated by the magnetic field pulse which varies slowly in amplitude across the sample. The pattern consists of $\nu$ regions where $\vec{M}$ has repeatedly reversed its original direction. The location of the boundaries between the regions reveals the energy required for the switching.
Owing to the internal clock provided by the precession of $\vec{M}$ about the intrinsic anisotropy fields, the boundaries also correspond to well defined times $t_{\nu}$ at which the switching occurred. These times exclude ultrafast dissipation claimed in pulsed laser excitation. But, amazingly, the dissipation of the spin angular momentum increases strongly after the first switch, exposing the opening of a new dissipation channel, which we associate with transfer of energy and angular momentum from the uniform magnetization precession mode to higher spin wave modes [10]. Such delayed dissipation explains why experiments [1-4] with only one precessional switch cannot detect the enhanced dissipation.

Prior to the field pulse, the magnetization of the film, $\vec{M}$, is oriented along the easy direction which we assume to lie in the $x y$ plane of the film, along the $x$ axis. Now, the sample is excited by the magnetic field pulse generated with a bunch of highly relativistic electrons $(28 \mathrm{GeV})$ from the linear accelerator. The electron beam travels along $z$, perpendicular to the film plane, and has a cross section of $9 \times 6 \mu \mathrm{m}(\mathrm{FWHM})$. As an electron bunch traverses the metallic film, its electric field is screened at the fs-time scale. The magnetic field $H^{p}$ penetrates the film. It is oriented perpendicular to the beam axis, resembling the familiar circular field generated by a straight current carrying wire $\vec{H}^{p}=I(t)[-y / r, x / r, 0] /(2 \pi r)$. Its strength is proportional to the electric current, $I(t)$, and decreases as $1 / r$, where $r=\sqrt{x^{2}+y^{2}}$ is the distance from the beam center at $x=y=0$. The distribution of the electrons along $z$ is Gaussian with a variance of $\sigma_{z}=630 \mu \mathrm{m}$ in the laboratory frame. This amounts to a FWHM duration of the Gaussian magnetic field pulse of $\tau=\sqrt{2 \pi} \sigma_{z} / c=$ $5.26 \mathrm{ps}$, where $c$ is the speed of light. The torque exerted 
by $\vec{H}^{p}$ on the magnetic moments $\vec{m}$ is $|\vec{T}|=\left|\vec{m} \times \vec{H}^{p}\right|=$ $m H_{y}^{p}$. The angle $\beta$ of the precession of the magnetic moments out of the film plane during the field pulse is calculated with the LLG equation using the magnetic anisotropies and the Gilbert damping obtained in separate FMR experiments [11]. $\beta$ lies in the range $10^{\circ}-25^{\circ}$ in the present experiments.

At the end of the field pulse, $\vec{M}$ is now in a nonequilibrium position, tending to relax into one of the two stable directions of the uniaxial material. In this process of relaxation, $\vec{M}$ precesses around the dominant demagnetizing field $\vec{H}^{\text {demag }}$ generated along the $z$ axis by the out-of-plane rotation of $\vec{M}$ during excitation plus much weaker fields due to the magnetic anisotropies. In this precession, a large angle $\theta=\left[90^{\circ}-\beta\right]$ is enclosed between $\vec{H}^{\mathrm{demag}}$ and $\vec{M}$, distinguishing it from that in FMR where $\theta$ is very small. In the large angle precession, the in-plane component $M_{x}$ oscillates periodically between the two easy directions. The damping of the precession establishes the dissipation of spin angular momentum, causing $\vec{M}$ to spiral back into the plane of the film until it can no longer overcome the anisotropy barrier imposed by the uniaxial, in-plane crystalline anisotropy energy $K_{u}$. Then, in the last phase, $\vec{M}$ oscillates about the in-plane uniaxial anisotropy field $H_{x}^{\text {ani }}=2 K_{u} / M$ until it comes to rest in either the initial direction along $x$, or the direction opposite to it.

If $K_{\perp}$ is the energy density of the total perpendicular anisotropy, the Zeeman energy density deposited in the spin system by the magnetic field pulse is given by

$$
E=K_{\perp} \sin ^{2} \beta .
$$

The energy $E\left(\beta_{1}\right)$ to induce the first switch is given by the energy $K_{u}$ to surmount the anisotropy barrier and the damping loss in the precession of $\vec{M}$ about $H^{\text {demag }}$ by $\varphi=$ $90^{\circ}$ to reach the anisotropy barrier. After that, the magnetization relaxes into the new direction in the last phase of the switching without consuming any additional energy. To switch $\vec{M}$ back, a higher energy $E\left(\beta_{2}\right)$ is needed to account for the damping loss in the additional precession by $\Delta \varphi=$ $180^{\circ}$. Each additional switching requires an energy increment $\Delta E_{\nu}=E\left(\beta_{\nu}\right)-E\left(\beta_{\nu-1}\right)$ to compensate for the damping loss in the $\nu$ th large angle precession by $\Delta \varphi=$ $180^{\circ}$. This is a truly ideal situation to measure the dissipation development in a precession enclosing a large angle $\theta$ between the magnetization and the magnetic field. The boundaries along which $\vec{M}$ has switched are contour lines of constant Zeeman excitation energy $E\left(\beta_{\nu}\right)=$ const.

The contour lines $E=$ const can be calculated from $\beta \propto$ $x / r^{2}=$ const, meaning that the precession about the intrinsic anisotropy fields can be neglected while the magnetic field pulse lasts. It applies in the parameter space of the present experiment. One obtains

$$
\left(\frac{x-a_{\nu}}{a_{\nu}}\right)^{2}+\left(\frac{y}{a_{\nu}}\right)^{2}=1 .
$$

The contour lines are thus circles of radius $a_{\nu}$ whose origin is shifted by $\pm a_{\nu}$ on the $x$ axis.

Figures 1 and 2 display examples of experimental switching patterns obtained with ultrathin films premagnetized along the easy axis parallel to the $x$ axis. It is evident that the experiment verifies the circular contour lines predicted by (2).

We used single crystalline bcc Fe films grown epitaxially on a GaAs(001) surface, protected by a 10 monolayer (ML) Au capping layer. The films have been characterized by FMR (yielding $g=2.09$ ) and other techniques [11]. The FMR linewidth is found to be independent of film thickness and increases linearly with FMR frequency from 9 to $70 \mathrm{GHz}$, corresponding to a Gilbert damping parameter $\alpha=0.004$. Up to 10 switches induced by a single electron bunch can be distinguished, as opposed to at most 4 switches in previous experiments with thicker Co films [8]. The constants $a_{\nu}$ are obtained by fitting circles to
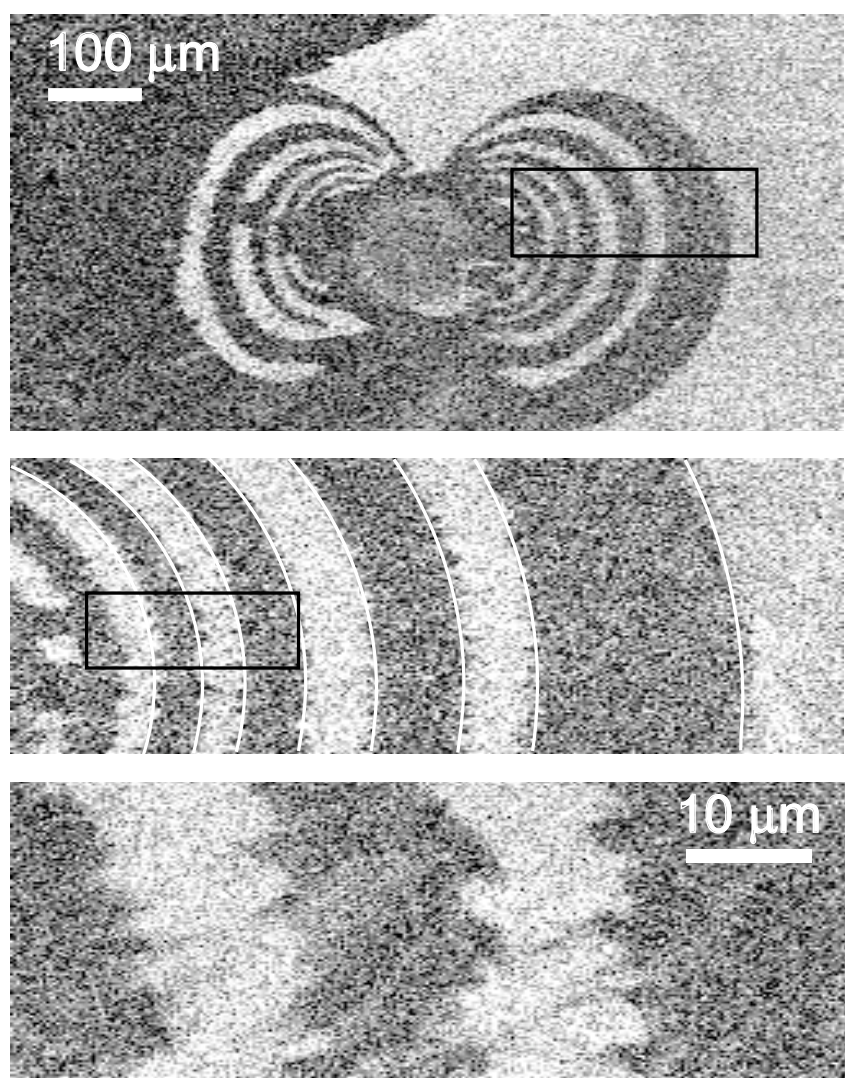

FIG. 1. Magnetic pattern generated with a single electron bunch in a $15 \mathrm{ML} \mathrm{Fe} / \mathrm{GaAs}(001)$ epitaxial bcc Fe film. The magnetic image is obtained by SEMPA after sputtering off the capping layer of $10 \mathrm{ML}$ Au. Prior to the field pulse, $\vec{M}$ is aligned horizontally to the right, shown in light gray. The regions were $\vec{M}$ has switched to the left are shown as dark gray. On the left and lower left side, the pattern is disturbed by motion of domain walls after exposure. In the center, a large spot due to beam damage appears. The framed part is shown at greater magnification in the middle with the fitted circles and at the bottom at still larger magnification exposing zigzag domain boundaries. 

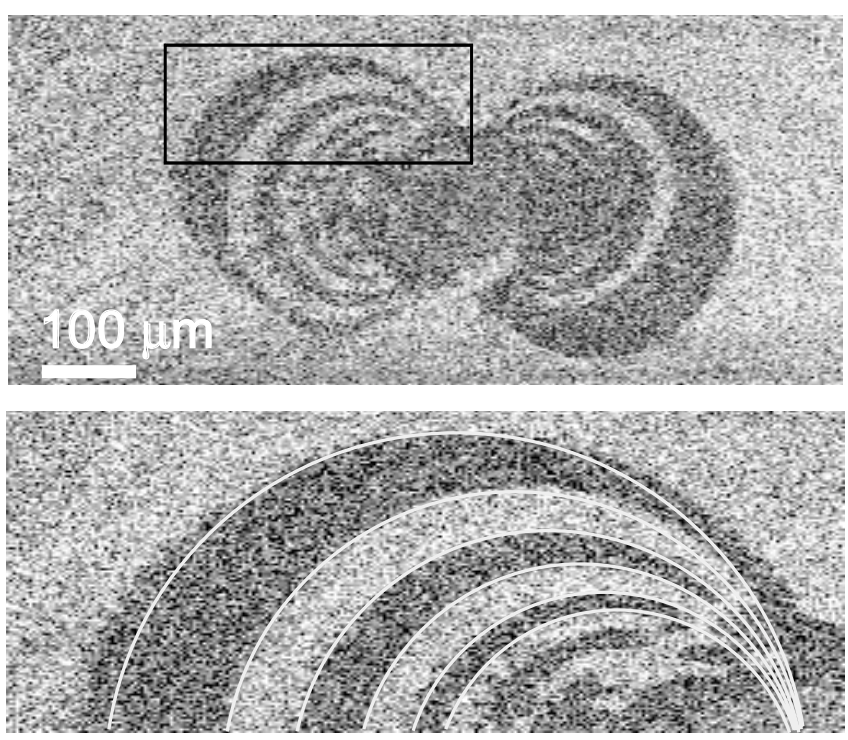

FIG. 2. Magnetic pattern generated with a single electron bunch in a $10 \mathrm{ML} \mathrm{Au} / 10 \mathrm{ML} \mathrm{Fe} / \mathrm{GaAs}(001)$ epitaxial Fe film, otherwise as in Fig. 1. No after pulse motion of domain walls occurred in this sample, but the pattern is less regular than with 15 ML.

the patterns as indicated in the figures. With the $10 \mathrm{ML}$ films, we observed 6 transitions of $\vec{M}$ at circle diameters $2 a_{1}, \ldots, 2 a_{6}=292,227,200,172,159$, and $139 \mu \mathrm{m}$, respectively. With the $15 \mathrm{ML}$ films, we determined 8 transitions at circle diameters $2 a_{1}, \ldots, 2 a_{8}=325,270$, $238,209,185,167,152$, and $139 \mu \mathrm{m}$, respectively. The accuracy of the $2 a_{\nu}$ is estimated to be $\pm 1 \mu \mathrm{m}$. It is not possible to quantify the location of the higher switches that are certainly present but appear to be fragmented.

The pattern of Fig. 1 is produced with an electron bunch of charge $Q=1.73 \mathrm{nC}$ in a $15 \mathrm{ML}$ Fe film with $H^{\text {ani }}=$ $4.72 \times 10^{4} \mathrm{~A} / \mathrm{m}$ and $H^{\mathrm{demag}}=128 \times 10^{4} \mathrm{~A} / \mathrm{m}$. The pattern of Fig. 2 is generated with $Q=2.1 \mathrm{nC}$ in a $10 \mathrm{ML} \mathrm{Fe}$ film. The thinner film exhibits a larger uniaxial anisotropy field of $H^{\text {ani }}=8.21 \times 10^{4} \mathrm{~A} / \mathrm{m}$ but a smaller $H^{\text {demag }}=$ $109 \times 10^{4} \mathrm{~A} / \mathrm{m}$. The magnetic patterns have been imaged 12 weeks after exposure of the samples to the field pulse by sputtering away the capping layers of $10 \mathrm{ML} \mathrm{Au}$ and then imaging the direction of $\vec{M}$ with scanning electron microscopy with polarization analysis (SEMPA, [12]). $\vec{M}$ is either parallel (light gray) or antiparallel (black) to the horizontal easy direction.

One type of problem encountered in determining the contour lines (2) is due to rugged zigzag transitions between regions of opposite $\vec{M}$. Such zigzag domain walls are displayed with high spatial resolution in the bottom section of Fig. 1. The switching leads initially to unfavorable "head-on" directions of $\vec{M}$ when a contour line runs perpendicular to the $x$ axis. As noted before [8], the headon transitions relax later into the longer but more favorable zigzag domain walls. The location of the switching transition is the average over the zigzag walls.
A second type of uncertainty arises from the fact that the samples are soft magnetic with a coercivity of $1-2 \mathrm{kA} / \mathrm{m}$ only. This means that domains may easily shift, e.g., in accidental magnetic fields. Apparently, domain wall motions occurred after exposure and deformed the left side of the pattern of Fig. 1, while on the right side the pattern appears to be undisturbed.

A third problem is the damage caused by the high energy electron bunch in the sample [13]. With the semiconducting GaAs substrate, the damage is larger compared to metallic buffer-layer substrates used in prior experiments $[8,9]$. The damage may be attributed to the electric field $E^{p}=c B^{p}$ running perpendicular the magnetic field $\vec{B}^{p}$ of the pulse. $E^{p}$ is not rapidly screened in a semiconductor, resulting in electrostrictive deformation of the GaAs template responsible for the uniaxial anisotropy. The permanent beam damage is delineated by a halo around the location of beam impact at $r \leq 50 \mu \mathrm{m}$. Although the halo is below the distances of the measurable switching events, it cannot be excluded that the magnetic anisotropy is affected transiently even at larger distances by the electrostrictive shock of the template.

With the $10 \mathrm{ML} \mathrm{Fe}$ film, the domain pattern is less regular than with the $15 \mathrm{ML}$ film. This must be due to larger local variations of the magnetic properties in the thinner film at the length scale of $100 \mu \mathrm{m}$ as the irregularities repeat themselves in different exposures.

If the energy given by Eq. (1) required for the onset of a new switch is plotted in units of $K_{u}$ vs the angle $\varphi$ of precession of $\vec{M}$, one obtains the universal switching diagram shown in Fig. 3. However, the experimental data depend somewhat on film thickness, as apparent at larger $\varphi$. Hence the diagram is not quite universal. In the first switch, $\vec{M}$ precesses by $\varphi=90^{\circ}$ only, and the switching energy $E / K_{u}$ is only slightly larger than 1 , compatible with a Gilbert damping parameter of $\alpha=0.005 \pm 0.005$. The experimental accuracy is thus insufficient to see any difference to the FMR damping $\alpha=0.004$. Yet already with the second switch, the additional precession by $180^{\circ}$ requires much more energy than that resulting from FMR damping. The loss in the higher switches grossly exceeds the dissipation expected from FMR (solid line) with both types of film.

The increase of the energy loss after the first switch shows that dissipation of spin angular momentum increases with time. Such delayed dissipation is characteristic for the Suhl instability [14], which is the transfer of energy from the uniform precession mode with wave vector $k=0$ to higher spin wave modes with $k \neq 0$. The transfer of energy, induced by nonlinear interactions owing to $H^{\text {demag }}$ and $H^{\text {ani }}$, takes time because the numbers of excited nonuniform spin waves grow exponentially with time. A quantitative theory for the dissipation caused by the Suhl instability has been developed recently [10]. In the inset of Fig. 3 we show simulations for an area of $1 \times 1 \mu \mathrm{m}$ of the 10 and 15 ML Fe films with a respective pulse ampli- 


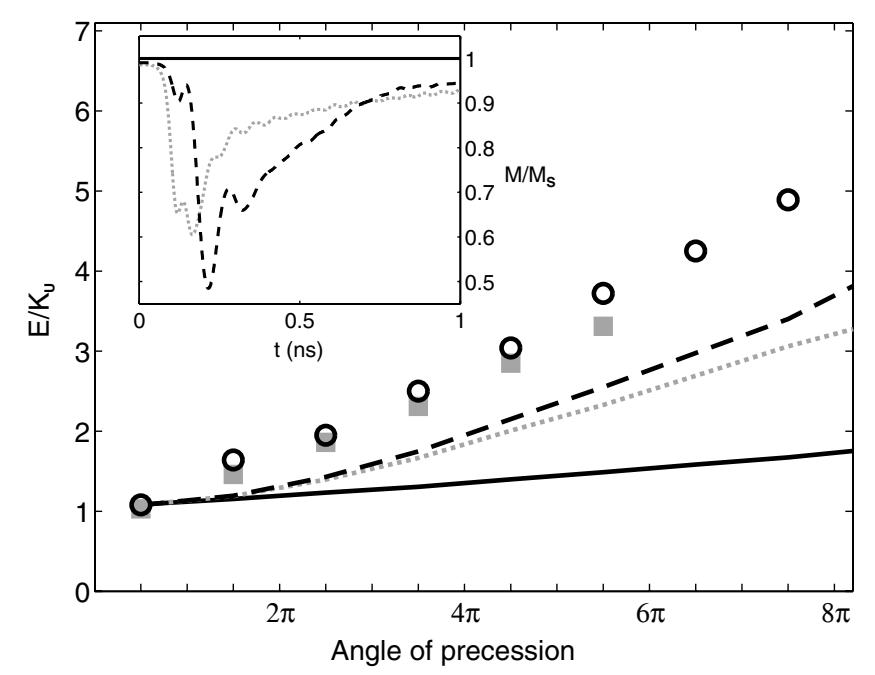

FIG. 3. Energy deposited in the spin system in units of the uniaxial in-plane anisotropy constant $K_{u}$ vs the precession angle $\varphi$. Data points are for $10 \mathrm{ML} \mathrm{Fe}$ (squares) and $15 \mathrm{ML} \mathrm{Fe}$ (circles). The simulations are with the Gilbert damping $\alpha=$ 0.004 and no magnon scattering (solid line), and for $10 \mathrm{ML} \mathrm{Fe}$ (dotted line) and $15 \mathrm{ML} \mathrm{Fe}$ (dashed line) including magnon scattering. The inset shows the relative saturation magnetization $M(t) / M_{s}(0)$, where $t$ is the time after an exciting field pulse of amplitude $0.24 \times 10^{6} \mathrm{~A} / \mathrm{m}$ for $10 \mathrm{ML} \mathrm{Fe}$ (dotted line) and $0.175 \times 10^{6} \mathrm{~A} / \mathrm{m}$ for $15 \mathrm{ML} \mathrm{Fe}$ (dashed line). Without magnon scattering (solid line) we have $M(t) / M_{s}=1$.

tude that completes the first switch. The magnetic field of the exciting pulse is homogeneous to better than $\Delta H^{p} / H^{p}=\Delta r / r<1 \%$ in this area. The simulation thus demonstrates the decrease of the space averaged order parameter $M / M_{s}$ with time in a homogeneous excitation: $M / M_{s}$ decreases sharply, $\approx 50 \mathrm{ps}$ after the field pulse, and recovers slowly through spin lattice relaxation of the spin waves. Now, from LLG simulations, we know the time $t_{\nu}$ after the field pulse at which the last change of sign of $M_{x}$ occurs. Therefore, we know the moment in time at which the energy consuming part of the switch $\nu$ is terminated. For the $15 \mathrm{ML}$ Fe film, we obtain $t_{1}, \ldots, t_{8}=40,115,155$, $195,235,270,310$, and 360 ps, respectively. Large dissipation is observed only after the first switch. This agrees with the 50 ps delay seen in the development of spinwave scattering. Furthermore, the fluctuations of $M / M_{s}$ in time and space manifest themselves through increasingly random switching as the angle $\varphi$ grows. Another characteristic of the Suhl instability concerns the film thickness. To conserve energy and momentum, the effective scattering of the uniform mode requires the excitation of low energy spin waves. The phase space for such low energy, long wavelength modes decreases with film thickness, and this explains the experiment as well as the simulation both showing smaller dissipation as the number of ML is reduced. Hence it is very likely that the Suhl instability contributes significantly to the dissipation observed in the experiment.
However, as apparent from Fig. 3, the simulations fall short by a factor 2 to fully account for the observed damping. Surface roughness is known to contribute to the damping. However, the detailed analysis based on [15] shows that the surface roughness measured on the present films [11] is not sufficient to explain the observations. Furthermore, it should show up in FMR as well. We therefore have to conclude that additional, so far unknown, relaxation mechanisms must be active in large angle precession of the magnetization as well.

SLAC is supported by the Office of High Energy and Nuclear Physics, the experimental program of the SSRL authors by the Office of Basic Energy Sciences, both of DOE. We wish to thank R. Iverson, C. Field, and G. J. Collet for their assistance in preparing and carrying out the sample exposure.

*Present address: BESSY, Albert-Einstein-Strasse 15, 12489 Berlin, Germany.

${ }^{\dagger}$ Electronic address: stohr@slac.stanford.edu

${ }^{\ddagger}$ Present address: University of Regensburg, Department of Physics, D-93040 Regensburg Germany.

[1] H. W. Schumacher, C. Chappert, P. Crozat, R. C. Sousa, P. P. Freitas, J. Miltat, J. Fassbender, and B. Hillebrands, Phys. Rev. Lett. 90, 017201 (2003).

[2] J. P. Nibarger, R. Lopusnik, and T. J. Silva, Appl. Phys. Lett. 82, 2112 (2003).

[3] T. Gerrits, H. A. M. van den Berg, J. Hohlfeld, L. Bar, and T. Rasing, Nature (London) 418, 509 (2002).

[4] S. Kaka and S.E. Russek, Appl. Phys. Lett. 80, 2958 (2002).

[5] W. K. Hiebert, G. E. Ballentine, and M. R. Freeman, Phys. Rev. B 65, 140404(R) (2002).

[6] G. P. Zhang and W. Hübner, Phys. Rev. Lett. 85, 3025 (2000).

[7] C. H. Back, D. Weller, J. Heidmann, D. Mauri, D. Guarisco, E. L. Garwin, and H. C. Siegmann, Phys. Rev. Lett. 81, 3251 (1998).

[8] C. H. Back, R. Allenspach, W. Weber, S. S. P. Parkin, D. Weller, E. L. Garwin, and H. C. Siegmann, Science 285, 864 (1999).

[9] I. Tudosa, C. Stamm, A. B. Kashuba, F. King, H. C. Siegmann, J. Stohr, G. Ju, B. Lu, and D. Weller, Nature (London) 428, 831 (2004).

[10] A. Yu. Dobin and R.H. Victora, Phys. Rev. Lett. 90, 167203 (2003).

[11] R. Urban, G. Woltersdorf, and B. Heinrich, Phys. Rev. Lett. 87, 217204 (2001); Georg Woltersdorf, Ph.D. thesis, Simon Fraser University, 2004.

[12] Robert J. Celotta and Daniel T. Pierce, Science 234, 333 (1986).

[13] Heating effects are negligible: the supersonic heat wave from the point of impact travels with only $1 \mu \mathrm{m} / \mathrm{ns}$ [9].

[14] H. Suhl, J. Phys. Chem. Solids 1, 209 (1957).

[15] A. Yu. Dobin and R.H. Victora, Phys. Rev. Lett. 92, 257204 (2004). 